Transport and Communications Science Journal, Vol. 71, Issue 1 (01/2020), 27-36

Transport and Communications Science Journal

\title{
A STUDY ON MEMORY DATA RETENTION IN HIGH-TEMPERATURE ENVIRONMENTS FOR AUTOMOTIVE
}

\section{Dao Thanh Toan}

Faculty of Electrical-Electronic Engineering, University of Transport and Communications, No 3 Cau Giay Street, Hanoi, Vietnam.

\section{ARTICLE INFO}

TYPE: Research Article

Received: 17/11/2019

Revised: 28/01/2020

Accepted: 30/01/2020

Published online: 31/01/2020

https://doi.org/10.25073/tcsj.71.1.4

${ }^{*}$ Corresponding author

Email: daotoan@utc.edu.vn

\begin{abstract}
The automotive memory devices especially work in high-temperature because they are located close to engine, exhaust units; those require high reliable operation and long-life data retention in high-temperature environments. This paper reports on the investigation of memory data retention of a nano-organic material-based nonvolatile memory in high-temperature environments. The decay of memory state current was theoretically presented as a stretched-exponential law. By fitting the measured currents to the stretched-exponential equation at different temperatures, the activation energy of decay and acceleration factor was deduced, which allows to predict the device performance at high-temperature environment like in a car. The method presented in study can be applied to estimate the memory data retention at high-temperature for other car memories.
\end{abstract}

Keywords: Automotive memory, high-temperature operation, stretched-exponential, car memory reliability 
Transport and Communications Science Journal, Vol. 71, Issue 1 (01/2020), 27-36

\section{INTRODUCTION}

A non-volatile memory is one of the most important electronic devices used in modern cars to store op-code or data for artificial intelligence in autonomous driving solutions [1]. Particularly, as shown in Fig. 1, the automotive memory circuits work especially in the high-temperature which can be from several tens to a hundred degree Celsius because of their locations close to engine, exhaust etc [2]. Those require high reliable operation and long-life data retention in high-temperature environments. Under the standard of ISO/TS 16949, the operational temperature of automotive non-volatile requires being more than $100{ }^{\circ} \mathrm{C}$; for example, the Fujisu memory can be worked at $125^{\circ} \mathrm{C}$ as shown in Fig. $1[3,4]$.

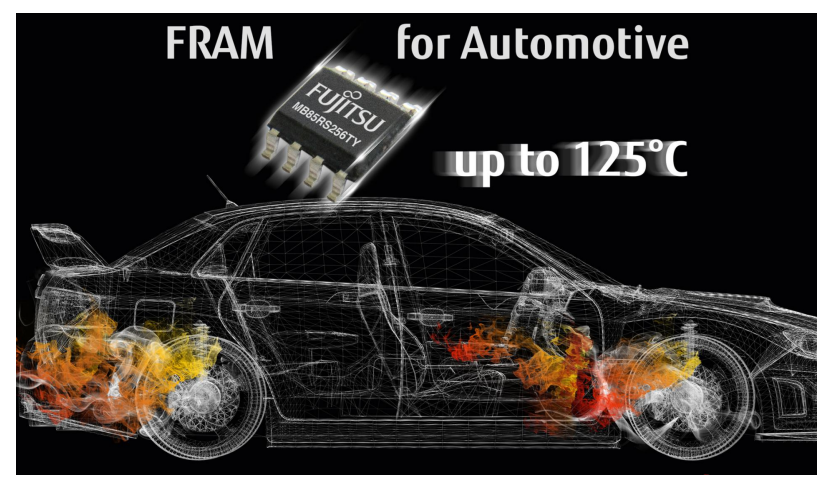

Figure 1. Non-volatile memory operating in high-temperature environments in a modern car [3].

In current non-volatile technology for automotive, a FET structure is widely used $[4,5]$ where the " 0 " or " 1 " are coded based on different values of the FET threshold voltage $\left(V_{\text {th }}\right)$. In such memory, instabilities of data storage were interpreted due to mobile charge carriers in the channel become trapped in the semiconductor or in the gate dielectric or at the active interface (so-called defects) under applying a gate voltage during the device operation [6]. The trapped charges deplete the channel, leading to $V_{\text {th }}$ change in memory device. However, the $V_{\text {th }}$ is indirectly obtained by extracting from the transfer curve. Overall, it is not very practical to examine the long-life data retention characteristics via the $V_{\text {th }}$ of the memory in high-temperature environments like in a car.

Recently, our university group has introduced a low-cost, easy-to-fabrication non-volatile FET memory fabricated from the environment-friendly nano-organic materials as shown in Fig. 2 [7] that is highly potential to use in automotive. This paper presents a progress which includes the following contributions:

- The stretched-exponential equation for the drain current $\left(I_{\mathrm{D}}\right)$ instead of the $V_{\text {th }}$ at high-temperature was derived for charge trap non-volatile memory.

- Through fitting with experimental data obtained in our University group, activation 
Transport and Communications Science Journal, Vol. 71, Issue 1 (01/2020), 27-36

energy of memory decay $\left(E_{\mathrm{a}}\right)$ and the long-term retention at different temperatures were realized to examine the applicability for the car.

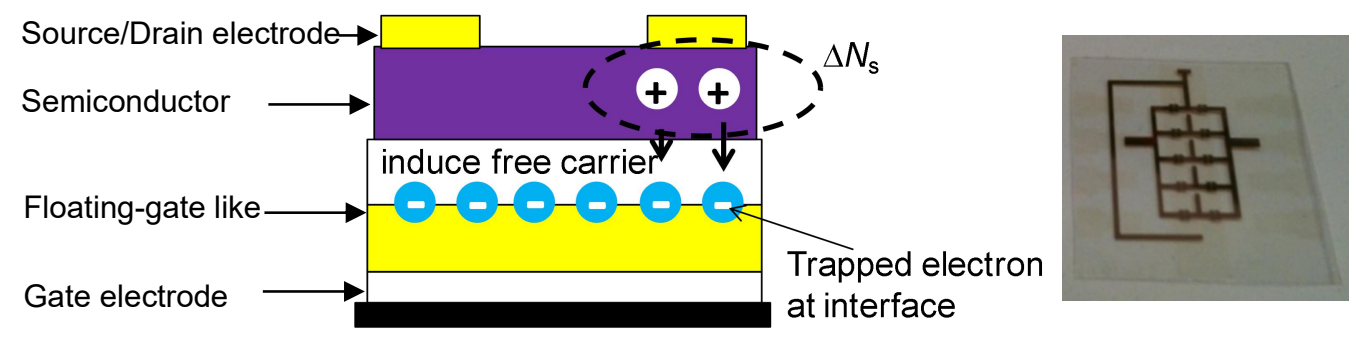

Figure 2. (left) Illustrational structure and interfacial trapped electron in memory and (right) photo of memory taken just after fabrication [7].

\section{DERIVATION OF STRETCHED-EXPONENTIAL EQUATION FOR MEMORY}

Under applying a gate bias during programming or erasing or reading memory, the mobile carriers were diffused and trapped at defect states, resulting in change in the free carriers in the FET channel. The rate creation of defect states can be described with the change in the density of free carrier $\left(\Delta N_{\mathrm{s}}(\mathrm{t})\right)$ as following relationship $[6,8]$ :

$$
\frac{d \Delta N_{s}}{d t}=-A * D(t) * \Delta N_{S}(t)
$$

where $t$ is time, $A$ is a proportionality constant, $D(\mathrm{t})$ is the diffusion coefficient, which is modelled by a dispersive diffusion coefficient given by $[9,10]$ :

$$
D(t)=D_{0} *(\omega t)^{-\alpha}
$$

In which $D_{0}$ is the microscopic diffusion constant, $\alpha$ is the temperature dependent dispersion parameter and $\omega$ is an attempt frequency for diffusion. After adding Eq. (2), Eq. (1) can be expressed as

$$
\frac{d \Delta N_{S}}{d t}=-A * D(t) * \Delta N_{S}(t)=-A * \Delta N_{S}(t) * D_{0} *(\omega t)^{-\alpha}
$$

Solving Equation (3)

$$
\begin{aligned}
& \frac{1}{\Delta N_{s}(t)} d \Delta N_{s}=-A D_{0} *(\omega t)^{-\alpha} d t \\
& \int \frac{d \Delta N_{s}}{\Delta N_{s}(t)}=-A D_{0} \int(\omega t)^{-\alpha} d t
\end{aligned}
$$


Transport and Communications Science Journal, Vol. 71, Issue 1 (01/2020), 27-36

$$
\operatorname{Ln}\left|\Delta N_{s}(t)\right|=-A D_{0} \frac{\left(\omega^{t}\right)^{-\alpha+1}}{\omega(-\alpha+1)}+\operatorname{Ln}\left|\Delta N_{s}(0)\right|
$$

Where $\Delta N_{s}(0)$ is the the initial free carrier density

$$
\operatorname{Ln}\left|\frac{\Delta N_{S}(t)}{\Delta N_{S}(0)}\right|=-\frac{A D_{0}}{\omega(-\alpha+1)}(\omega t)^{-\alpha+1}
$$

When $\tau$ is presented as:

$$
\begin{gathered}
\tau=\sqrt[-\alpha+1]{\frac{\omega(-\alpha+1)}{A D_{0}}\left(\frac{1}{\omega^{-\alpha+1}}\right)} \\
\text { Or } \frac{1}{\tau^{-\alpha+1}}=\frac{A D_{0}}{\omega(-\alpha+1)} \omega^{-\alpha+1}
\end{gathered}
$$

Eq. (7) can be expressed as

$$
\begin{gathered}
\operatorname{Ln}\left|\frac{\Delta N_{S}(t)}{\Delta N_{S}(0)}\right|=-\left(\frac{t}{\tau}\right)^{-\alpha+1} \\
\frac{\Delta N_{S}(t)}{\Delta N_{S}(0)}=\exp \left\{\left(-\frac{t}{\tau}\right)^{-\alpha+1}\right\}
\end{gathered}
$$

Let $\beta=1-\alpha$, it has:

$$
\frac{\Delta N_{s}(t)}{\Delta N_{s}(0)}=\exp \left\{\left(-\frac{t}{\tau}\right)^{\beta}\right\}
$$

Where $\tau$ is the characteristic time constant

During retention time process, the relaxation of the trapped electrons causes the change in density of free carrier, leading to change of $V_{\text {th,ON }}$ as:

And

$$
\Delta N_{S}(0)-\Delta N_{S}(t)=e \frac{\Delta V_{t h, O N}}{C_{i}}=e \frac{V_{t h, O N}(0)-V_{t h, O N}(t)}{C_{i}}
$$

$$
\Delta N_{S}(0)-\Delta N_{S}(\infty)=e \frac{\Delta V_{t h, O N}}{C_{i}}=e \frac{V_{t h, \text { ON }}(0)-V_{t h, \text { ON }}(\omega)}{C_{i}}
$$

where $\Delta N_{\mathrm{s}}(0), \Delta N_{\mathrm{s}}(\mathrm{t})$, and $\Delta N_{\mathrm{s}}(\infty)$ are the free carrier density in the channel at the initial $(\mathrm{t}=0)$, a certain $(\mathrm{t}=\mathrm{t})$, and at the infinite $(\mathrm{t}=\infty)$ times, respectively; e is the elementary charge, and $C_{\mathrm{i}}$ is the capacitance per unit area of the gate dielectric.

From (13) and (14):

$$
\frac{\Delta N_{S}(0)-\Delta N_{S}(t)}{\Delta N_{S}(0)-\Delta N_{S}(\infty)}=\frac{V_{t h, O N}(0)-V_{t h, O N}(t)}{V_{t h, O N}(0)-V_{t h, O N}(\infty)}
$$

By assuming that at the infinite time, the trapped electrons completely are removed, i.e.,

$$
\Delta N_{s}(\infty)=0
$$


$\frac{V_{t h, O N}(0)-V_{t h, O N}(t)}{V_{t h, O N}(0)-V_{t h, O N}(\infty)}=\frac{\Delta N_{S}(0)-\Delta N_{S}(t)}{\Delta N_{S}(0)-\Delta N_{S}(\infty)}=\frac{\Delta N_{S}(0)-\Delta N_{S}(t)}{\Delta N_{S}(0)}=1-\frac{\Delta N_{S}(t)}{\Delta N_{S}(0)}=1-\exp \left\{\left(\frac{t}{\tau}\right)^{-\beta}\right\}$

It has the equation for the shift in $V_{\text {th }}$ of the ON state:

$\Delta V_{t h, O N}=V_{t h, O N}(t)-V_{t h, O N}(0)=\left\{V_{t h, O N}(\infty)-V_{t h, O N}(0)\right\}\left\{1-\exp \left\{\left(\frac{t}{\tau}\right)^{-\beta}\right\}\right\}$

In the memory device, the $V_{\text {th }}$ is extracted from the transfer curve that is measured by sweeping the gate voltage from positive to negative regions and the transfer curve of a FET can be described in the linear regime using the equation [6]:

$$
I_{D}=\frac{W * \mu * C_{i}}{L}\left(V_{G}-V_{t h}\right) V_{D} \text {, with }\left|V_{G}-V_{t h}\right|>V_{D}
$$

where, $L$ and $W$ are the channel length and channel width, and $\mu$ is the FET mobility.

By using the boundary condition that at the infinite time, $I_{D}(\infty)=0$, thus from (19)

$$
V_{t h}(\infty)=V_{G}
$$

By replacing (20) into (18):

$$
V_{t h, O N}(t)-V_{t h, O N}(0)=\left\{V_{G}-V_{t h, O N}(0)\right\}\left\{1-\exp \left\{\left(\frac{t}{\tau}\right)^{-\beta}\right\}\right\}
$$

$=>$

$$
\begin{aligned}
& 1-\frac{V_{t h, O N}(t)-V_{t h, O N}(0)}{V_{G}-V_{t h, O N}(0)}=\exp \left\{\left(\frac{t}{\tau}\right)^{-\beta}\right\} \\
& \frac{V_{G}-V_{t h, O N}(t)}{V_{G}-V_{t h, O N}(0)}=\exp \left\{\left(\frac{t}{\tau}\right)^{-\beta}\right\}
\end{aligned}
$$

On the other hand, the equation (19) at the time of initial and t can be expressed as

$$
I_{D, O N}(0)=\frac{W * \mu * C_{i}}{L}\left\{V_{G}-V_{t h, O N}(0)\right\} V_{D}
$$


And

$$
I_{D, O N}(t)=\frac{W * \mu * C_{i}}{L}\left\{V_{G}-V_{t h, O N}(t)\right\} V_{D}
$$

From (25) and (24), it can be obtained:

$$
\frac{I_{D, O N}(t)}{I_{D, O N}(0)}=\frac{V_{G}-V_{t h, O N}(t)}{V_{G}-V_{t h, O N}(0)}
$$

By replacing (26) into (23), the stretched-exponential relationship of the $I_{\mathrm{D}}$ at the ON state can be realized:

$$
\frac{I_{D, O N}(t)}{I_{D, O N}(0)}=\exp \left\{\left(\frac{t}{\tau}\right)^{-\beta}\right\}
$$

After programming, the drain current changes from $I_{\mathrm{D}, \mathrm{OFF}}$ to $I_{\mathrm{D}, \mathrm{ON}}$. In the used memory device, the measurements indicate that $I_{\mathrm{D}, \mathrm{OFF}}$ do not change very much [7]. Thus, investigation of retention time of the memory is simply equal to observation of the change in the $I_{\mathrm{D}, \mathrm{ON}}$ obeyed Eq.(27).

\section{FITTING CURRENT AT DIFFERENT TEMPERATURES}

The current values of the memory at different temperatures from 25 to $100{ }^{\circ} \mathrm{C}$ were measured at gate voltage of $0 \mathrm{~V}$ and a drain voltage of $-2 \mathrm{~V}$ with a Keithley 4200 semiconductor characterization system in dry nitrogen. The measurement method has been presented in detail in recent work [7]. It notes here that the highest temperature is $100{ }^{\circ} \mathrm{C}$ because it makes sure that the organic materials in memory are not effective by their glass temperatures. Figure 3 shows the typical normalized currents at the haft-life time those were obtained from experiments (black square curves) and the stretched-exponential equation (red-square curves) in an Origin lab. Experimental data fitted very well with the stretched-exponential equation, suggesting that the developed eq. (27) is very suitable for memory. Inset of each sub-figure in Fig. 3 shows the mathematical parameters of fitting where the $\tau$ and $\beta$ are denoted by a or b respectively. As the result, the $\tau$ and $\beta$ value can be obtained at each temperature value. Subsequently, the activation energy of the relaxation can be deducted from Arrhenius plot in Fig. 4a followed equation below [11]:

$$
\tau=\omega^{-1} \exp \left(\frac{E_{a}}{k T}\right)
$$

Where, $\mathrm{k}$ is the Boltzmann constant $\left(8.62 \times 10^{-5} \mathrm{eV} \mathrm{K}^{-1}\right)$, and $\omega^{-1}$ the frequency pre-factor. $E_{\mathrm{a}}$ and $\omega$ were estimated to be $0.98 \mathrm{eV}$ and $1.56 \times 10^{8} \mathrm{~s}^{-1}$, respectively. 
Transport and Communications Science Journal, Vol. 71, Issue 1 (01/2020), 27-36
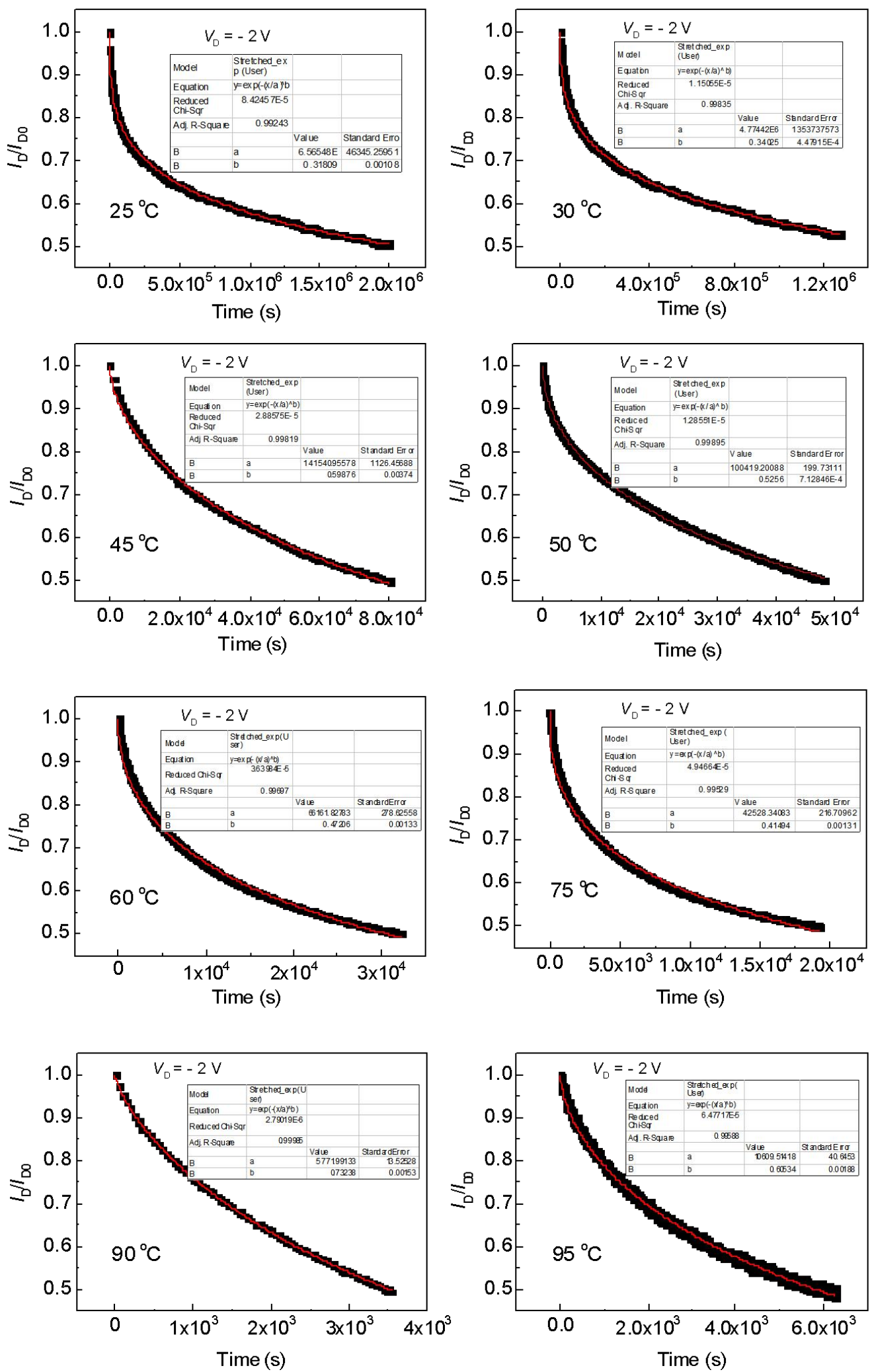

Figure 3. Normalized On-state drain current decay at different temperature. Experimental data are presented by black colour and red colour curves represent fitted data using stretched equation. 
Transport and Communications Science Journal, Vol. 71, Issue 1 (01/2020), 27-36

The $\beta$ exhibited to be linearly dependent on temperature as $\beta=T / T_{0}-\beta_{0}$, where $T_{0}$ is the characteristic temperature. The dispersion parameter $\beta$ presents the width of the distribution of $E_{\mathrm{a}}$ as $k T_{0}$ [9-12]. As presented in Fig. $4 \mathrm{~b}, T_{0}$ and $\beta_{0}$ were extracted to be 227.27 $(\mathrm{K})$ and 0.94 , respectively. The distribution width of $E_{\mathrm{a}}$ was estimated to be $0.02 \mathrm{eV}$. Ideally, $\beta$ $=T / T_{0}$, but in literature data usually shows existence of $\beta_{0}$.
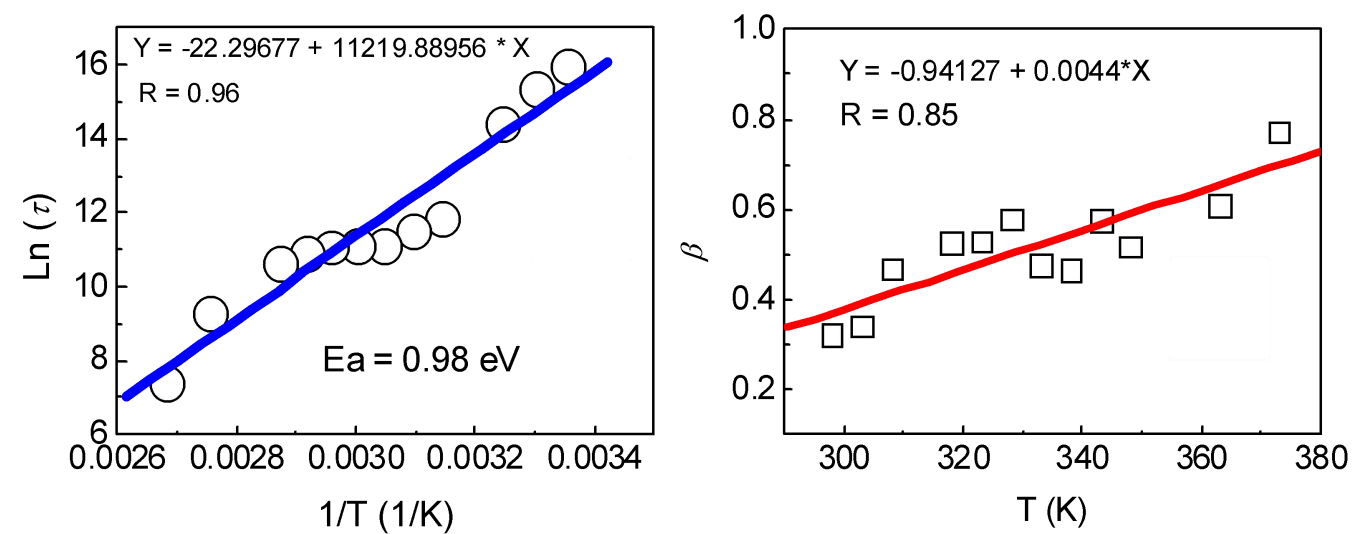

Figure 4. $\operatorname{Ln}(\tau)-1 / T$ (top) and $\beta-T$ (bottom) plots. $\tau$ and $\beta$ were deduced from the fitting curves of experimental $I_{D}$ at different temperatures and stretched exponential equation.

\section{ESTIMATION OF DATA RETENTION AT HIGH-TEMPERATURE}

An acceleration method has been used to estimate long-term retention time of memory $[11,12]$. The acceleration factor $(A F)$ is defined as the ratio of the time at base temperature $T_{1}$ $\left(\mathrm{t}\left(T_{1}\right)\right)$ to that at elevated temperature $T_{2}\left(\mathrm{t}\left(T_{2}\right)\right)[11]$ :

$$
A F=\frac{t\left(T_{1}\right)}{t\left(T_{2}\right)}=\frac{\exp \left(\frac{E_{a}}{k T_{1}}\right)}{\exp \left(\frac{E_{a}}{k T 2}\right)}=\exp \left\{\frac{E_{a}}{k}\left(\frac{1}{T_{1}}-\frac{1}{T_{2}}\right)\right\}
$$

Thus, life time at room temperature can be extracted by equation below [11]:

$$
\mathrm{t}\left(T_{1}\right)=A F^{*} \mathrm{t}\left(T_{2}\right)
$$

In current memory, the $\mathrm{AF}$ at $100{ }^{\circ} \mathrm{C}$ was found to be 1505 . The predicted retention times of data storage at difference temperatures using acceleration method are shown in Table 1. Overall, the retention time reduces with increasing operational temperature. For example, at $25{ }^{\circ} \mathrm{C}$, retention time of data storage can be almost 10 years; meanwhile, it drops to only 0.5 year at $100{ }^{\circ} \mathrm{C}$. Thus, in order to use in car environment at highest reliability, it is necessary to package the memory by temperature insulating materials. 
Transport and Communications Science Journal, Vol. 71, Issue 1 (01/2020), 27-36

Table 1. Predicted life-time of data retention time at different temperatures.

\begin{tabular}{llllllll}
\hline Operated temperature $\left({ }^{\circ} \mathbf{C}\right)$ & $\mathbf{2 5}$ & $\mathbf{5 0}$ & $\mathbf{6 0}$ & $\mathbf{7 0}$ & $\mathbf{8 0}$ & $\mathbf{9 0}$ & $\mathbf{1 0 0}$ \\
\hline Retention time (year) & 10 & 5 & 4 & 3 & 1.2 & 0.6 & 0.5 \\
\hline
\end{tabular}

Table 2. Quick comparison among current and other memories.

\begin{tabular}{llll}
\hline & Memory in this study & $\begin{array}{l}\text { Fujisu memory for } \\
\text { car [3] }\end{array}$ & $\begin{array}{l}\text { Memory used in } \\
\text { Mercedes car [4] }\end{array}$ \\
\hline $\begin{array}{l}\text { Maximum operated } \\
\text { temperature }\left({ }^{\circ} \mathrm{C}\right)\end{array}$ & 100 & 125 & 160 \\
Cost & low & high & high \\
Fabrication & easy & complicated & complicated \\
\hline
\end{tabular}

\section{CONCLUSIONS}

Based on the theoretical and experimental studies in collaboration with analysis, the following conclusions can be drawn:

- The change in the $V_{\text {th }}$ after programming can be converted to observation of the change in the $I_{\mathrm{D}}$ obeying a stretched-exponential distribution, which can help to avoid effect of scanned voltages on the stored electrons in the floating gate.

- By measuring the degradation of the current at different temperatures and fitting to the stretched-exponential distribution, the $E_{\mathrm{a}}$ and $A F$ can be estimated and forward to use predict the long-term retention time of data storage at high-temperatures in car. This method can be applied to other car memory technologies.

- It requires for the current memory reported here to be encapsulated before using high-temperature environments in a car since the encapsulated materials can help to reduce the temperature affecting directly on the memory. Even the retention time of data storage at high-temperature of the memory is not very high, but it has some positive things in comparison with other automotive memory technologies as shown in Table 2. Thus, it is highly motivated to literature researchers to keep going on study this memory in order to commercialize in automotive industry.

\section{ACKNOWLEDGMENT}

This research is partly funded by Vietnam Minister of Education and Training (MOET) under grant number B2019-GHA-03. 
Transport and Communications Science Journal, Vol. 71, Issue 1 (01/2020), 27-36

\section{REFERENCES}

[1] J. R. Jameson, Towards Automotive Grade Embedded RRAM, 48th European Solid-State Device Research Conference, 2018, 5 pages. https://doi.org/10.1109/ESSDERC.2018.8486890

[2] F. Pieri et al., Limits of sensing and storage electronic components for highreliable and safety-critical automotive applications, International Conference of Electrical and Electronic Technologies for Automotive, 2017, 7 pages. https://doi.org/10.23919/EETA.2017.7993219

[3] https://www.fujitsu.com/uk/about/resources/news/press-releases/2017/feeu-20170202-1.html_ (accessed 16 Nov 2019).

[4] https://www.flashmemorysummit.com/English/Collaterals/Proceedings/2017/20170808_FT12_H uonker.pdf_(accessed 30 October 2019).

[5] Cuong Manh Tran, Electron trapping mechanism in a multi-level organic FET memory using lithium-ion-encapsulated fullerene as the floating gate, Transport and Communication Science Journal, 70 (2019) 193-200. https://doi.org/10.25073/tcsj.70.3.5

[6] H. Sirringhaus, Reliability of organic field-effect transistors, Adv. Mater. 21 (2009) 3859-3873.

[7] T. T. Dao et al., Low switching voltage, high-stability organic phototransistor memory based on a photoactive dielectric and an electron trapping layer, Organic Electronics (2019) 105505 (in press). https://doi.org/10.1016/j.orgel.2019.105505

[8] D. M.de Leeuw et al,. Dynamics of Threshold Voltage Shifts in Organic and Amorphous Silicon Field-Effect Transistors, Adv. Mater. 19 (2007) 2785. https://doi.org/10.1002/adma.200602798

[9] H.H.Choi, W. H. Lee, K.Cho, Bias-stress-induced charge trapping at polymer chain ends of polymer gate-dielectrics in organic transistors, Adv. Funct. Mater. 22 (2012) 4833. https://doi.org/10.1002/adfm.201201084

[10] H.H. Choi et al., Decoupling the bias-stress-induced charge trapping in semiconductors and gate-dielectrics of organic transistors using a double stretched-exponential formula, Adv. Funct. Mater. 23 (2013) 690-696. https://doi.org/10.1002/adfm.201201545

[11] T.N. Ng, B. Russo, A.C. Arias, Degradation mechanisms of organic ferroelectric field-effect transistors used as nonvolatile memory, J. Appl. Phys. 106 (2009) 094504. https://doi.org/10.1063/1.3253758

[12] K. Lee et al., Memory effect in organic transistor: controllable shifts in threshold voltage, Chem. Phys. Lett. 551 (2012) 105. https://doi.org/10.1016/j.cplett.2012.09.022 